\section{Innovación y desarrollo de nuevos productos. Caso Alaska Mousse D’Onofrio}

\author{
Innovation and development of new products. Case Alaska \\ Mousse D'Onofrio
}

\begin{abstract}
RESUMEN
La innovación es todo cambio que genera valor para el consumidor y para la sociedad, por tal motivo es considerado pilar de crecimiento en la industria de helados, tal es el caso de Alaska Mousse de D'Onofrio. El objetivo del artículo es entender los atributos relevantes de la categoría de los helados de fruta a través de las actitudes y el comportamiento del consumidor de la marca que cada vez se vuelve más racional y selectivo a la hora de planificar sus gastos; para ello se ha utilizado la metodología cualitativa exploratoria a través de cuatro Focus Group, realizando una prueba en mesa del nuevo producto Alaska Mousse, donde las reacciones ante la prueba fueron muy positivas, así tenemos que los atributos relevantes para el mencionado target en primer lugar está la naturalidad, en segundo lugar, el sabor, sobre todo que sea cercano al sabor real de la fruta, sin percepción de saborizantes intensos o artificiales y con un nivel de dulce equilibrado principalmente. Cabe indicar que desde la aplicación de la estrategia de innovación de producto y siguiendo el proceso de Desarrollo de nuevos productos se incrementaron las ventas inclusive en temporada de invierno.
\end{abstract}

Palabras Clave: Innovación; Nuevos productos; Target Alaska Mousse; Naturalidad.

\begin{abstract}
Innovation is any change that generates value for the consumer and for society, which is why it is considered a pillar of growth in the ice cream industry, such is the case of D'Onofrio's Alaska Mousse. The objective of the article is to understand the relevant attributes of the fruit ice cream category through the attitudes and behavior of the consumer of the brand, who is becoming increasingly rational and selective when planning their spending; for this purpose the exploratory qualitative methodology has been used through four Focus Groups, The reactions to the test were very positive, so we have that the relevant attributes for the mentioned target in the first place is the naturalness, secondly, the taste, especially that it is close to the real taste of the fruit, without perception of intense or artificial
\end{abstract}

\section{Sonia Hermelinda Ramón} Musaurieta soniaramon14@hotmail.com Nestlé Perú S.A. Lima, Perú

Presentado: 06/11/2020 - Aceptado: 31/03/2021 - Publicado: 17/06/2021

(C) Los autores. Este artículo es publicado por la revista Gestión en el Tercer Milenio de la Facultad de Ciencias Administrativas Universidad Nacional Mayor de San Marcos. Este es un artículo de acceso abierto, distribuido bajo los términos de la licencia Creative Commons Atribución 4.0 Internacional (CC BY 4.0) [https://creativecommons.org/licenses/by/4.0/deed.es] que permite el uso, distribución y reproducción en cualquier medio, siempre que la obra original sea debidamente citada de su fuente original. 
flavorings and with a balanced sweetness level mainly. It should be noted that since the application of the product innovation strategy and following the new product development process, sales have increased, even in the winter season.

Keywords: Innovation; New products; Target Alaska Mousse; Naturalness.

\section{INTRODUCCIÓN}

El helado ha sido, desde siempre, un producto del verano que quedaba restringido a la tradición de la temporada de calor, por ello para que el consumo de helados no sea estacional, la estrategia de innovación de producto es determinante para el crecimiento de la industria de helados, tal es el caso de la marca de helados de fruta Alaska Mousse D'Onofrio, el cual fue desarrollado e industrializado entendiendo las actitudes, necesidades y comportamiento del consumidor de este segmento, que cada vez se vuelve más racional y selectivo a la hora de planificar sus gastos. Como siguiente paso, para medir la respuesta del mencionado target se realizó la prueba en mesa del prototipo de Alaska Mousse durante la etapa de desarrollo, donde las reacciones ante la prueba fueron muy positivas para el consumidor: los componentes de hielo y crema del prototipo cubre sus necesidades de "antojo" en cualquier época del año, así como la naturalidad del producto fue considerado como atributo relevante.

En ese sentido, es importante iniciar con las definiciones de los autores de los términos de innovación, creatividad y nuevos productos así dar un panorama claro del concepto de Innovación de producto con el desarrollo de nuevos productos. Una vez aclarado los conceptos, la gestión del proceso de innovación es decir para su aplicación en las industrias será mediante el proceso de desarrollo de nuevos productos. Para ello se explican cada una de las ocho etapas del proceso.

Durante la etapa de desarrollo y prueba de producto se diseña un prototipo de producto el cual resume las prioridades de los consumidores target de Alaska, al momento de elegir un helado de fruta, entendiendo los atributos relevantes que valoran como son la naturalidad principalmente. El prototipo fue Alaska mousse, tiene 2 componentes: crema suave tipo mousse de fresa en el interior y capa de hielo de fruta fresa en la superficie de helado, el cual fue testeado a grupos de consumidores meta.

De acuerdo a lo mencionado, los resultados del focus group demuestran que la innovación de Alaska por los 2 componentes que tiene el producto, hielo y crema de fruta en la parte interior fue percibida de manera muy positiva y ha logrado apropiarse de los momentos divertidos del verano y del invierno, por su capacidad de refrescar y también de indulgencia.

\section{Objetivo}

El presente artículo tiene como objetivo entender al consumidor de este segmento como eje central para desarrollar la estrategia de innovación y la gestión del proceso de desarrollo de nuevos productos en el mercado de frutas, caso del helado de fruta: Alaska mousse fresa, también cabe resaltar que los nuevos productos conllevan a nuevas soluciones y sobre todo brinda diversidad a la vida de los consumidores y clientes. El gran aporte a la sociedad es incentivar el empleo de productores locales de nuestros recursos naturales como son las frutas regionales oriundas de la sierra y de la selva, como el aguaymanto, fresa, lúcuma, maracuyá, mango, entre otros.

\section{Argumentación}

Tal como se determinó previamente, al momento de mencionar el objetivo del artículo, se focaliza al consumidor como eje principal para desarrollar la estrategia de innovación de producto, por tal motivo es necesario iniciar el estudio explicando los conceptos de innovación. Adair (1992) afirma "Innovar significa literalmente producir o introducir algo nuevo, alguna idea, algún método o instrumento nuevos. En particular combina dos procesos que se superponen: tener ideas nuevas y ponerlas en marcha" (p.IX). 
Los nuevos productos son el elemento indispensable y vital de una organización. Una compañía obtiene nuevos productos de dos maneras. Una es mediante la adquisición, es decir al comprar una empresa, una patente o una licencia para comercializar el producto de alguien más. La otra manera es mediante sus propias actividades de desarrollo de nuevos productos. (Kotler y Armstrong, 2017, p. 270)

Así cuando hablamos de productos nuevos nos referimos a productos que son auténticos, también pueden ser proyectos para realizar alguna mejora o modificar un producto y servicios que ya existen en su cartera, asociadas a nuevas marcas que desarrolle la compañía gracias a contar con una estructura de investigación y desarrollo. Sobre la innovación Schnarch (2009) dice "la innovación implica necesariamente dos procesos: tener ideas nuevas y ponerlas en marcha" (p. 47). Finalmente, para entender la relevancia de la innovación se debe considerar como el eje central de la estrategia de un negocio o emprendimiento (Kuczmarki, 1997).

Ahora vamos a definir qué se entiende por producto de acuerdo con diferentes autores.

Como cualquier bien que se puede ofrecer a un mercado para su atención, adquisición, uso o consumo, y que podría satisfacer un deseo o una necesidad. Los productos no sólo son bienes tangibles, como automóviles, computadoras o teléfonos celulares. Los productos incluyen objetos físicos, servicios, eventos, personas, lugares, organizaciones, ideas o mezclas de ellos. (Kotler y Armstrong, 2017, p. 232)

Una gestión eficiente del proceso de innovación y desarrollo de nuevos productos centrado en el consumidor es sistemática. Kotler y Armstrong (2017) explican que son ocho las etapas por el cual pasa un proceso de desarrollo de nuevos productos, desde cómo se generan y luego se depuran las ideas, luego el desarrollo y prueba del concepto, después el desarrollo del marketing en cuanto a la estrategia, el análisis de negocio, desarrollo de productos, mercado de pruebas y finalmente la etapa de comercialización. Para un entendimiento mejor de este proceso se explican cada una de las etapas que exponen Kotler y Armstrong (2017):

\section{Etapa 1 - Generación de las ideas}

Diferentes metodologías son aplicadas para generar nuevas ideas así identificar entre la colección de ideas aquellas que muestren alto potencial en entregar resultados para alcanzar los objetivos. Son tres puntos relevantes que la alta gerencia debe establecer: el alcance, el mercado y los objetivos del producto; también ser específicos en cuanto a las modificaciones o mejoras de productos existentes o copiar productos de los competidores. Las fuentes para la generación de ideas de nuevos productos pueden ser de: clientes, proveedores, competidores, científicos, empleados, fuerza de ventas, visibilidad, la alta gerencia, miembros del canal etc.

\section{Etapa 2 - Depuración de ideas}

Existe un comité de nuevos productos en el cual se filtran las ideas ganadoras que sean coherentes con la estrategia y los objetivos de innovación y/o renovación.

En la prueba llamada de concepto se realiza una evaluación de la idea de producto, para después crear un prototipo, en este punto se recibe la percepción de cómo reaccionan los consumidores del mercado meta mediante descripciones, versiones o representaciones visuales del prototipo propuesto.

\section{Etapa 3 - Desarrollo y prueba de conceptos}

Para esta tercera etapa la idea seleccionada como ganadora se desarrolla de manera eficiente para convertirse en un concepto de producto. "El concepto es una versión detallada de la idea del producto expresada en términos significativos para el cliente o consumidor" (Kotler y Armstrong, 2017, p. 275). Durante la prueba de concepto se evalúan conceptos de productos nuevos a los futuros consumidores para determinar el grado de atracción. La presentación de los conceptos a los consumidores puede ser de forma simbólica, real física (prototipo) o potencial.

\section{Etapa 4 - Desarrollo de estrategias de marketing}

Es la fase en el cual se diseña la estrategia de mercadeo inicial para el lanzamiento del 
producto que calificó mejor en las pruebas. En primer lugar, podemos establecer la descripción del mercado meta, la propuesta de valor, los objetivos de ventas, participación de mercado y la rentabilidad. También la marca, el diseño y la calidad de empaque, las etiquetas; en segundo lugar, las declaraciones vinculadas a las estrategias preliminares de precio, promoción, distribución y presupuesto de marketing para un periodo de un año, en tercer lugar, se consideran las ventas proyectadas a largo plazo, la utilidad objetivo y la estrategia de la mezcla de marketing.

\section{Etapa 5 - Análisis de negocios}

Esta etapa implica realizar un costeo preliminar llamado también de factibilidad financiera, que implica la revisión de costos (costos fijos y variables, recursos, inversiones, mano de obra entre otros), proyecciones de ventas y utilidades de un nuevo producto para determinar si esos resultados cumplen las claves de éxito del proyecto y satisfacen los objetivos de la compañía. Si es así el producto pasará a la etapa de desarrollo.

\section{Etapa 6 - Desarrollo de productos}

Es la conversión del concepto del producto en un bien físico tangible de tal manera que así garantizamos que la idea del producto se convierta en oferta factible de mercado. El departamento de investigación y desarrollo creará y probará una o más versiones físicas del concepto del producto, así como diseñar un prototipo que satisfaga e interese a los clientes y consumidores de una manera ágil dentro de los costos presupuestados.

Un nuevo producto debe contar con las características funcionales requeridas y transmitir además los rasgos psicológicos deseados.

\section{Etapa 7 - Mercado de prueba}

Etapa de desarrollo de un nuevo producto en la cual el producto y el programa de marketing propuestos se introducen a pruebas en ambientes de un mercado real. Lo que se persigue en esta prueba es introducir el producto de manera limitada, teniendo un programa de comercialización de tal manera poder evaluar la respuesta de los clientes potenciales, cuáles serían las alternativas de las estrategias y determinar cómo se integrarían a la mezcla de mercadeo. Las ciudades o lugares que son seleccionadas como característica principal deben simular las condiciones del mercado para el nuevo producto, tanto en lo demográfico, como en rutina de compra y distribución.

Se recomienda que los lugares designados para la prueba deben estar sin influencia de los medios de comunicación, porque podría suceder que las pruebas de productos pueden atraer a otro tipo de consumidores que no son el objetivo.

\section{Etapa 8 - Comercialización}

En base al marketing de prueba, la gerencia tendrá la información que necesita para tomar una final y correcta decisión sobre el lanzamiento del nuevo producto. La etapa de comercialización o lanzamiento donde el producto nuevo llega al mercado, se debe dar publicidad interna y externa, entrenamiento tanto a la fuerza de ventas como a empresas encargadas de su distribución y a los consumidores finales.

\section{MÉTODOS}

Para el presente estudio se ha utilizado la técnica Cualitativa exploratoria a través de dinámicas grupales como son los grupos focales o focus group, para enfocarse en explorar motivaciones, detectar percepciones, valores, actitudes y creencias de los consumidores los cuales aportan información valiosa del potencial de un concepto. El objetivo es presentar el prototipo de la innovación de producto como es el helado Alaska mousse fresa al público objetivo y asegurar que su desempeño satisface, encuentren valor e interés a los consumidores.

Los grupos focales, focus group, son una técnica de recolección de datos mediante una entrevista grupal semiestructurada, la cual gira alrededor de una temática propuesta por el investigador.

Público Objetivo: Personas de Nivel Socioeconómico (NSE) B2/C1 entre 18 y 30 años, no rechazadores de helados y conozcan la marca Alaska.

Muestra: Para la muestra se distribuyeron en 4 grupos, el primer grupo predomina el consumo de helado Alaska, el segundo grupo predomina el consumo de helados Artika 
(principal competidor), el tercer grupo consume Alaska como otras alternativas y el cuarto grupo predomina el consumo de sustitutos. Para un mejor entendimiento de los grupos de enfoque, se muestra la Tabla 1.

Tabla 1

Distribución de los Grupos de enfoque

\begin{tabular}{cl}
\hline $\mathbf{N}^{\circ}$ & Descripción \\
\hline $\mathbf{1}$ & Predomina el consumo de Alaska \\
$\mathbf{2}$ & Predomina el consumo de helados Artika \\
$\mathbf{3}$ & Consumen Alaska como otras alternativas \\
$\mathbf{4}$ & Predomina el consumo de sustitutos \\
\hline
\end{tabular}

Nota. Adaptado del Informe cualitativo Alaska Paths (1), adaptado de Ipsos (2018), pp. 3-52.

\section{RESULTADOS}

El desarrollo del prototipo de Alaska mousse como innovación de producto junto al concepto de otras innovaciones, con una comunicación de naturalidad, las reacciones de la prueba en mesa fueron muy positivas, el $95 \%$ quedaron muy satisfechos de la prueba sensorial de producto, porque percibieron un mayor sabor real de la fruta, como evidencia los consumidores sintieron la presencia las semillas o pepitas de la fresa, por lo cual se consideró una buena estrategia de la marca la comunicación de naturalidad. Adicionalmente, combinar el hielo y crema, lo hace complacer el antojo, pero con un nivel de dulzor equilibrado. El relleno del mousse se percibe cremoso y ligero, el $30 \%$ lo asoció al yogurt que es considerado saludable, la combinación de ambos componentes en el caso del Alaska mousse, la crema interior de fresa con la capa de hielo de fruta fresa es como comerse un postre para los consumidores de Alaska.

La investigación fue presentada por la empresa de investigación de mercado Ipsos mediante un informe cualitativo escrito, que es un documento formal sistematizado, sintetizado, que demuestra y concluye los hallazgos del estudio.

\section{DISCUSIÓN}

El estudio se realizó con el público objetivo de la marca Alaska para validar la investigación y obtener información directa de la percepción de la innovación en el producto: Alaska mousse.
Con todas las indagaciones, se ha detectado que para la elección de un helado de fruta los consumidores de este segmento basan principalmente su elección en el sabor, un sabor cercano al sabor real de la fruta, sin percepción de saborizantes intensos y artificiales, y con un nivel de dulce equilibrado, luego está el precio, la marca y la disponibilidad.

Las razones profundas detrás de los hábitos y percepciones de los consumidores del segmento fue saber que la naturalidad del producto se verifica sensorialmente, es decir con la prueba en el sabor natural y un color suave, no se revisa la etiqueta del producto para buscar la evidencia de naturalidad, validando así el prototipo de Alaska mousse al percibir las semillas de la fruta como evidencia de la fruta real de la fresa como atributo de naturalidad.

Sin embargo, los consumidores con una marcada preferencia por los helados Artika, llamados Artika lovers, principal competencia en el mercado de helados, si bien sintieron agrado por el producto, aún consideran que esta versión no llega a tener el nivel de naturalidad que ellos valoran. Algunos de ellos llegaron a percibir que podría empalagarlos y saturarlos, por lo que consideran que su consumo sería esporádico.

\section{CONCLUSIONES}

Con la metodología empleada podemos inferir que el consumidor vive constantemente modificando su conducta frente a la compra de productos o servicios. En la actualidad se vuelve relevante las tendencias saludables, es decir conseguir un equilibrio perfecto en el producto que sea rico y saludable. La denominación para los nuevos consumidores que representan el $19 \%$ del total de la población son los llamados healthy balancers.

La innovación de Alaska Mousse Fresa, es un gran lanzamiento para la marca en su camino hacia la naturalidad, se percibe mayor sabor real a fruta principalmente en el relleno que tiene semillas de la fresa, es cremoso y ligero, que lo asocian al yogurt, que es considerado saludable, al tener dos componentes es decir, un helado de hielo (Shell) y crema (Core) cumpliría con la expectativa no solo de refrescar sino al 
combinar hielo y crema lo hace complacer el antojo o postre en temporada de invierno.

Para el público objetivo de los consumidores de Alaska, los helados de hielo y fruta, si bien se orientan a saciar en mayor medida la sed y refrescar a quien lo consume, también tienen una cuota de indulgencia y búsqueda de placer por el hecho de ser helados, por lo cual comunicar masivamente salud en esta categoría no generará identificación ni atractivo.

El atributo más importante es el sabor en esta categoría, este deberá ser superior en la medida en que sea natural y lo más cercano a la fruta real, logrando mayor sensación de frescura, con el nivel adecuado de dulce y, de manera más secundaria, generando en la mente del consumidor la tranquilidad de estarse dando un gusto que no atentará tanto contra su salud.

El sabor natural, la naturalidad es valorada, muy cercano a las frutas reales es verificada, principalmente, al momento de degustar el producto, sin embargo, la comunicación de las Calorías también se percibe positivamente por ser bajas para un helado (menor a $100 \mathrm{ca}-$ lorías), pero no sería el driver de consumo de este producto.

El consumidor durante todo el año está buscando opciones más saludables dentro de la categoría "Alimentos" y sobre todo en verano cuando se preocupan más por su imagen. Alaska se ha posicionado como el helado de fruta, pero pierde relevancia si no hay innovaciones en el portafolio en especial en temporada de invierno donde el consumo es más por indulgencia, por lo cual innovaciones de ediciones limitadas podría ser una buena alternativa.
Se recomienda nuevos lanzamientos con innovaciones que generen atractivo no sólo en temporada de verano sino también en temporada de invierno, como pueden ser paletas con trozos grandes de fruta real con cobertura de Cacao, que evidencia naturalidad. Hay posibilidad de potenciar la naturalidad y, a su vez generar sentimientos de orgullo peruano, al lanzar innovaciones con frutas peruanas exóticas y la utilización de cacao de nuestras regiones.

Cobraría mayor diferenciación si se desarrolla en base a frutas peruanas oriundas de la sierra y selva, porque al hablar del origen busca conectar a nivel emocional desde un territorio que no está muy asociado a los helados (conectan con indulgencia y diversión), con potencial para acercar más al sabor real a frutas y flanquear las opciones de fruta de la competencia Artika.

\section{REFERENCIAS BIBLIOGRÁFICAS}

Adair, J. (1992). El reto gerencial de la innovación. Legis.

Ipsos. (2018). Informe cualitativo Alaska Paths. (Reporte n. ${ }^{\circ}$ ). 3-52.

Kotler, P., y Armstrong, G. (2017). Marketing. Pearson Educación de México.

Kuczmarski, T. (1997). Innovación, Estrategias de liderazgo para mercados de alta competencia (Traducido de la primera edición). McGraw-Hill Interamericana S.A.

Schnarch, A. (2009). Creatividad, innovación y marketing. Desarrollo de nuevos productos y empresas (5. ${ }^{\mathrm{a}}$ ed.). McGraw-Hill Interamericana S.A. 